\title{
Ueber Empis nitida Meig. und die ihr verwandten Arten.
}

Vom

Prof. Dr. H. Loew, Director a. D. in Guben.

Meigen hat Empis nitida in die Gattung Pachymeria gestellt. Ich habe mich bereits in Band VIII. der Wiener entomol. Monatsschrifı dahin ausgesprochen, dafs ihre nächsten verwandtschaftlichen Beziehungen diese Stellung als unzulässig erscheinen lassen. Ich komme hier nochmals auf diesen Punkt zurück, um meine Ansicht ausführlicher zu begründen und um diejenigen Arten, welche mit Emp. nitida einen geschlossenen Verwandtschaftskreis bilden, genauer zu besprechen.

Es ist ganz richlig, dafs Emp. nitida Merkmale besitzt, welche auf eine Verwandtschafi mil Pachymeria femorala und mit deren nächsien Verwandten hindeuten. Die gelrennten Augen des Männchens, die verdickten Hinterschenkel und die in Folge davon, wie bei Pach. femorata, an ihrer äufsersten Basis gekrümmten Hinlerschienen, so wie die verhältnifsmäfsige Kleinheit der Discoidalzelle sind die auffallendsten derselben. Diesen Merkmalen gegenüber stehen andere Merkmale, welche sie von Pach. femorala und deren nächsten Verwaudten wesentlich unterscheiden; dahin gehören die längeren Fühler, die grofse Sparsamkeit der Behaarung des Thoraxrückens, die an ilrem Ende etwas nach hinten gebeugte dritte Längsader, die sich gegen ihr Eude hin viel weniger erweiternde erste Hinlerrandszelle, vor allem aber der ganz abweichende Bau des Hinterleibs und der äufseren Genitalien; der männliche Hinterleib ist keineswegs wie bei Pach. femorala gegen sein Ende hin zugespitzt und gerade, sondern fast gleichbreit, am Ende abgerundet und abwärts gekrümmI; das Hypopygium ist in eigenthümlicher Weise vom Hinterleibe abgeschnürt und hildet eine plumpe, fast fufsförmige Masse; der Hinterleib des Weibchens ist nicht allmälig zugespitzt, sondern bis zum fünften Ringe hin vollkommen gleichbreit, von hier ab aber plölzlich sehr verschmälert und in eine lange Spitze auslaufend, welche wie dem vorderen Theile des Hinterleibs besonders angesetzl erscheint; die Anallamellen, welche bei Pach. 
femorala und ihren Verwandten auffallend kurz sind, haben eine sehr grofse Länge und sind sehr schmal.

Diese Merkmale, durch welche Emp. nitida sich von den ächten Pachymeria-Arten unterscheidet, finden sich bei einer Anzahl ihr nahe verwandter Arten, zum Theil in gesteigertem Maafse wieder, während diejenigen Merkmale, welche Emp. nitida den ächten Pachymeria-Arten ähnlich machen, bei diesen Arten allmälig immer mehr zurücktreten. Sie bilden mit Emp. nitida einen gut geschlossenen Verwandtschaftskreis, zu welehem wahrscheinlich auch Emp. tumida Meig. gehört. Mir sind von den in denselben gehörigen Arten bisher nur fünf bekannt geworden; diese sind: 1) nitida Meig., 2) monticola nov. sp., 3) truncata M eig., 4) lucida $\mathrm{Z}$ ett. und 5) longipennis nov. sp. -

1. Emp. nitida Meig. - Diese Art ist in ganz Mitteleuropa nirgends selten, auch über ganz Italien verbreitet; ob sie auch in anderen Theilen Südeuropa's vorkommt, weifs ich nicht; Herr Schiner schreibt ihr Faun. I. 110 irrthümlich Schweden als Vaterland zu, während sie bis jetzt dort noch nicht gefunden worden ist. Meigen's Angaben enthalten eine Ungenauigkeit, welche leicht unberechtigte $Z \mathbf{w}$ eifel an der Richtigkeit der Artbestimmung erwecken kann; er nennt nämlich die Flügel glasartig; so können sie aber nur bei dem Männchen genannt werden, keineswegs aber bei dem Weibchen, welches Meigen seiner Angabe nach doch ebenfalls gekannt hat; bei dem Weibchen haben sie an der Spize eine glasartig grane Färbung, welche gegen die Basis hin in eine deutliche Bräunung übergeht, welche bei recht ausgefärbten Exemplaren ziemlich intensiv ist. Ich habe in mehreren Sammlungen das Männchen unter der richtigen Bestimmung, das Weibchen aber als vermeintlich neue Art gefunden. Das wahre Sachverhältnifs hat schon vor Jahren Dr. Scholtz in den Schriften des schlesischen entomologischen Vereins auseinander gesetzt.

Zu den Merkmalen, welche Meigen angiebt, sind zur sicheren Kenntlichmachung der Art wenigstens noch folgende hinzuzufügen. Stirn bei beiden Geschlechtern breit. Fühler mit sehr langem und sehr schmalem dritten Gliede und mit sehr kurzem Griffel. Taster braunschwarz; Mundrand glänzend schwarz. Thorax dunkel aschgrau, die beiden Theile der Mittelstrieme und die Seitenstriemen braun; Prothoraxstigma schwarz; die äulsere Ecke der Schulterschwiele rothgelb. Die Behaarung des ganzen Körpers ohne Ausnahme schwarz, verhältnifsmäfsig kurz, auf dem Thoraxrücken sehr sparsam, auf den Seitenrand und auf die Interstitien zwischen der Mittelstrieme und den Seitenstriemen beschränkt; die Mittellinie, 
durch welche die Mittelstrieme halbirt wird, ist kahl. Dic Behaarung des glänzend schwarzen Hinterleibs ist bei dem Weibchen überall kurz, bei dem Männchen nur an den Seiten der vorderen Ringe ziemlich lang. Schenkel schwarz mil rothgelber Spitze; die hintersten auffallend verdickt, besonders bei dem Männchen, bei beiden Geschlechtern auf ihrer Unlerseite dicht mit kurzen schwarzen Borsten besetzt. Schienen rothgelb, zuweilen mehr braungelb, bei sehr dunkelgefärbten Exempl. zum gröfsten Theile dunkelbraun; die vorderen ohne eigentlicheStachelborsten; die hintersten von kräftigem Baue, in der Nähe der Basis am dicksten und an der äufsersten Basis selbst in ähnlicher Weise wie bei den ächten Pachymeria-Arten gebogen, oder eigentlich fast gebrochen, auf der Oberseite ungleich und sparsam behaart, auf der Unterseite bei dem Männchen von ganz überaus kurzen, senkrecht abstehenden Borstchen gewimpert, bei dem Weibchen mit minder abstehender, kurzer Behaarung von gewöhnlicher Beschaffenheit besetzt. Die Füfse sind an ihrer Wurzel wie die Schienen gefärbt, gegen ihr Ende hin geht die Färbung allmählig in das Schwarze über; bei den dunkelsten Exemplaren sind sie mit Ausnahme der gelbbraunen Wurzel fast ganz schwarz. Klauen und Pulvillen sind bei dem Weibchen nur von gewöhnlicher, bei dem Männchen aber von sehr auffallender Gröfse. Schwinger gelb, der Stiel gewöhnlich erheblich dunkler. Flügel ziemlich lang; alle Adern vollständig; die erste Submarginalzelle von mäfsiger Breite; dritte Längsader am Ende sanft nach hinten gebogen; der Vorderast derselben schief und ziemlich weit vor ihrem Ende angesetzt, so dafs die zweite Submarginalzelle eine ansehnliche Gröfse hat; die erste Hinterrandszelle verbreitert sich gegen ihr Ende hin nur sehr mäfsig; die Discoidalzelle ist klein und am Ende ziemlich schief, doch nicht ganz so schief als bei den ächten Pachymeria-Arten. Die Flügel des Männchens sind glasartig mit so aufserordentlich schwacher graulicher Trübung, dafs man dieselbe durchaus nicht bemerken könnte, wenn sie der Flügelwurzel nicht fehlte; die Hülfsader und alle Adern in der Nähe der Flügelwurzel sind gelb, die Costa sammt den drei ersten Längsadern dunkelbraun, die übrigen Adern mehr gelbbraun oder gelbbräunlich. Flügel des Weibchens glasartig grau, gegen die Basis hin deutlich und gewöhnlich stark gebräunt; alle Adern derselben dunkelbraun, nur in der Nähe der Wurzel zum Theil gelbbräunlich.

Emp. nitida fliegt in der norddeutschen Ebene bereits im ersten Drittel des Mai; ihre Körperlänge belrägt $2 \frac{1}{6}-2 \frac{1}{3}$ Lin., die Fliigellänge $2 \frac{1}{3}-2 \frac{1}{2} \mathrm{Lin}$.

2. Emp. monticola, nov. sp., fliegt in den Alpen im Juli und 
im Anfang des August. Ich habe sie zuerst im Jahre 1855 auf der Saualpe in Kärnthen gefunden, besitze aber auch Weibchen aus der Schweiz, welche zu Ende Juli auf der Bernina gefangen wurden. - Ich vermag diese Art durchaus auf keine der publizirten zurückzuführen und habe sie deshalb neu benennen müssen. Sie steht zu Emp. nitida in nahen verwandtschaftlichen Beziehungen, welche, soweit Meigen's Angaben einen Schlufs zulassen, denen ähnlich zu sein scheinen, in welchen die mir leider noch unbekannte Emp. tumida zu Emp. nitida steht. Meine Art für Emp. tumida zu halten, verbieten das gelbliche Prothoraxstigma und der aschgraue Hinterleib, welche Meigen der Emp. tumida zuschreibt, da bei meiner Art beide schwarz sind, und der letztere überdiefs überall einen lebhaften Glanz zeigt. Emp. montana ist ein Mittelglied zwischen Emp. nilida und Emp. truncata, deren letzterer sie fast noch näher steht.

Die Körperfarbe ist glänzend schwarz, doch sind Kopf und Thorax grau bestäubl. Die Stirn ist bei beiden Geschlechtern breit. Fühler schwarz; die beiden ersten Glieder mit sehr kurzer schwarzer Behaarung; das dritte Glied verhältnifsmäfsig lang, an der Basis breit, gegen das Ende hin allmälig verschmälert; der Griffel kurz aber schlank. Mundrand glänzend schwarz. Taster klein, gelb, mit etlichen ganz kurzen schwarzen Härchen. Rüssel kräftig; die Oberlippe eiwa so lang wie die Vorderschienen. Scheitel und Oberhälfte des Hinterkopfs mit kurzer, borstenartiger, schwarzer Behaarung. Thorax mit nur mäfsig dichter graulicher Bestäubung, welche die glänzend schwarze Grundfarbe desselben nicht ganz verdeckl; eine deutliche Slriemung ist auf dem Thoraxrücken nicht vorhanden, doch erscheinen in den meisten Richtungen die Interstitien gıauer als die Striemen; die sparsame und kurze schwarze Behaarung des Thoraxrückens ist auf die Seitenränder und auf die Interstitien zwischen der Millelstrieme und den Seitenstriemen beschränkt; die Mittellinie ist, wie bei Emp. nilida, völlig kahl. Der Prothorax ist unmittelbar über den Vorderhüften mit zarter, blafs fahlgelblicher Behaarung besetzt. Der Haarschirm vor den Schwingern ist bei den auf der Saualpe gefangenen Exemplaren aus ziemlich zarten fahlgelblichen Haaren gebildet; bei den Weibchen aus der Schweiz ist die Färbung dieser Haare theils fahlbräunlich, theils ziemlich dunkel fahlbraun, so dafs sie in manchen Richtungen braunschwarz erscheinen. Schildchen nur mit schwarzen Randborsten. Hinterleib bei beiden Geschlechtern glänzend schwarz, mit sehr zerstreuter, blafs gelblicher Behaarung, welche an den Seiten des männlichen Hinterleibs sehr lang und fast zollig, an denen des weibli- 
chen Hinterleibs ziemlich lang ist. Dieselbe gelbliche Färbung hat die kurze Behaarung des Hypopygiums, doch sind ihr auf dem zweiten Theile seiner Unterseite einige etwas längere schwarze Haare beigemengt. Das vorletzte Bauchsegment des männlichen Hinterleibs mit zwei nahe beieinander stehenden Zapfen. Hüften schwarz mit dünner graulicher Bestäubung; die Behaarung derselben fahlgelblich oder fast hell fahlbräunlich, die stärkeren Haare an den Hinterhüften und meist auch an der Spilze der Vorderhüften schwarz. Beine bräunlich kastanienroth, die Oberseite der vorderen Schenkel auf ihrer ersten Hälfte oft dunkler braun; die sehr kur»e Behaarung der Beine schwarz. Die Schenkel sind kräftig, die hintersten nur mäfsig verdickt und bei dem Männchen, wie es mir scheint, im Verhälınifs zur Körpergröfse ein wenig länger als bei dem Weibchen; die Vorderschenkel sind nur auf der zweiten Hälfte, die Mittel- und Hinterschenkel auf der ganzen Unterseite beborstet. Schienen fast ganz unbeborstet, die wenigen Borstchen derselben sehr schwach; die Hinterschienen gerade, am Ende ein wenig stärker als an der Basis. Füfse schlank, die vordersten gewöhnlich von der Basis aus, die andern wenigsleus gegen das Ende hin braunschwarz oder schwarz. Klauen und Pulvillen bei dem Männchen etwas gröfser als bei dem W'eibchen, aber verhältnifsmäfsig viel kleiner als bei dem Männchen der Emp. nilida. Flügel sehr lang, fast glasartig, mit wässriger, aber deutlicher bräunlichgrauer Trübung; Aderı dunkelbraun, in der Nähe der Basis bräunlichgelb; erste Submarginalzelle mälsig breit; dritte Längsader am Ende sanft nach hinien gebogen; Vorderast derselben ziemlich weil vor ihrem Ende angesetzt und die zweite Submarginalzelle deshalb verhältnifsmäfsig grofs; die erste Hinterrandszelle verbreitert sich gegen ihr Ende bin etwas mehr, als bei Emp. nitida; Discoidalzelle etwas gröfser, als bei dieser, am Ende mälsig schief. Körperlänge $2 \frac{1}{4}-2 \frac{7}{12}$ Lin., Flügellänge $2 \frac{1}{1} \frac{1}{2}-3 \frac{1}{3} \operatorname{Lin}$.

Anmerkung. Ich will hoflen, dafs ich die in der Schweiz gefangenen Weibchen nicht mit Unrecht zu Emp. monticola gezogen habe. Den Unterschied in der Färbung des Haarschirms vor den Schwingern habe ich schon oben hervorgehoben. Ich mufs hier noch bemerken, dal's bei den in der Schweiz gefangenen Weibchen die Hinterschenkel etwas stärker und etwas kürzer zu sein scheinen als bei den Weibchen, welche ich auf der Saualpe erbeutete.

3. Emp. Truncata Meig. - Ich bin zweifelhaft gewesen, ob ich diese Art als Emp. truncata Meig. oder Emp. Bistortae Meig. aufführen solle. Ich habe sie früher nicht für Emp. truncala zu 
halten gewagt, weil die von Meigen Thl. III. 31 gegebene Beschreibung dieser Art von Wiedemann nach einem portugisischen Weibchen der Hoffmannsegg'schen Sammlung angefertigt worden ist und hinsichtlich der Färbung der Flügeladern und der Schienen nicht recht auf unsere Art pafst. Das typische Weibchen befindet sich noch im Berliner Museum und die Ansicht desselben giebt mir die Gewifsheit, dafs es mit dem Weibchen unserer Art vollkommen einerlei ist und dafs die Wiedemann'sche Beschreibung hinsichllich derjenigen Merkmale, welche nicht auf unsere Art passen wollen, unricbtig ist. Die Anwendung des Namens Emp. truncata auf unsere Art ist also eine vollkommen sichere. Dasselbe läfst sich von der Anwendung des Namen Emp. Bistortae nicht sagen. Die Häufigkeit der Emp. truncata in Deutschland, das Zusammentreffen ihrer Flugzeit mit der Blüthezeit der Bistorta, welche sie mit ganz besonderer Vorliebe aufsucht, sowie das Zutreffen der hervorstechendsten der von Meigen der Emp. Bistortae zugeschriebenen Merkmale liefsen mich früher vermuthen, dafs unsere Art die von Meigen als Emp. Bistortae beschriebene sei. Ich hege auch noch jetzt dieselbe Vermuthung, obgleich ich bekennen mul's, dal's mehrere der Angaben, welche Meigen über Emp. Bistortae macht, nicht auf unsere Art passen. So beschreibt er den Rüssel als $1 \frac{1}{2} \mathrm{mal}$ so lang als der Kopf; da die in ihrer Länge unveränderliche Oberlippe bei unserer Art mehr als $1 \frac{1}{2} \mathrm{mal}$ so lang als der Kopf ist, so will seine Angabe auf unsere Art nicht wohl passen; ferner sagt er, dafs aus dem Hypopygium ein feiner Faden vorrage, bei unserer Art aber ist der Penis ganz eingeschlossen, so dafs seine Angabe sich auf unsere Art nur unter der Voraussetzung beziehen läfst, dal's er die beiden borstenartigen schwarzen Haare, welche sich am unteren Theile ihres Hypopygium finden, für einen feinen Faden angesehen habe; die Beine nennt er haarig, während man vermuthen möchte, dafs er dieselben bei einer Art wie die unsrige eher kahl genannt haben würde. - Wenn ich trotz diesen Widersprüchen in Emp. Bistorlae immer noch unsere Emp. truncaia vermuthe, so mufs ich doch dem letzteren Namen als dem allein vollkommen gesicherten entschieden den Vorzug geben; er wird, da er eben so alt ist, ihr auch bleiben müssen, wenn künftig mit Sicherheit nachgewiesen werden sollte, dafs Emp. Bistortae mit Emp. truncata identisch ist.

Emp. Iruncata ist glänzend schwarz; Kopf und Thorax sind graulich bestäubt. Augen bei beiden Geschlechtern getrennt; Stirn des Weibchens nur mäfsig breit; die des Männchens sehr verschmälert; sie hat bei beiden Gesehlechtern kurze Behaarung, welche gröfstentheils schwarz ist, nur weiter oben zum Theil eine fahl- 
gelbliche Färbung hal. Scheitel und Oberhälfte des Hinterkopfs mit borstenartiger schwarzer Behaarung; die Unlerhälfte des letzteren mit zarlerer und längerer, ganz bleich fahlgelblicher, fast schmutzig weifslicher Behaarung. Fühler schwarz; die beiden ersten Glieder mit kurzer schwarzer Behaarung; das dritte Glied lang und schmal; der Griffel kurz. Mundrand glänzend schwarz; Oberlippe etwas länger als die Vorderschienen; Taster braun, gewöhnlich mit gelberer Spitze, zuweilen ganz und gar schmutzig gelb, mit lichter Behaarung, nur an der Spitze auch mit etlichen kurzen schwarzen Härrhen. Die graue Bestäubung des Thorax verdeckt die glänzend schwarze Grundfarbe desselben nicht ganz, so dafs sich an den Brustseiten je nach der Betrachtungsweise veränderliche graue Flecken zeigen und dafs auf dem Rücken eine deutliche Striemung nicht wahrnehmbar wird, doch erscheinen in den meisten Richtungen die Striemen etwas dunkler als die Interstitien. Die kurze und sehr sparsame Behaarung des Thoraxrückens beschränkt sich auf den Seitenrand und auf die Interstitien zwischen der Mittelstrieme und den Seilenstriemen; die Mittellinie desselben ist, wie bei den vorhergehenden Arten, völlig kahl; der Prothorax ist unmittelbar über den Vorderbüften sparsam mit bleich fahlgelblicher, fast schmulzig weifslicher Behaarung beselzt und der Haarschirm vor den Schwingern hat ebenfalls eine fahlgelbliche, zuweilen fast hell fahlbräunliche Farbe. Der Hinterleib ist durchaus glänzend schwarz, mit zerstreuter fahlgelblicher Behaarung von verhältnifsmäfsig sehr grofser Länge besetzt, welche an den Seiten des männlichen Hinterleibs ein fast zottiges Ansehen hat. Die Behaarung des Hypopygiums hat zum allergröfsten Theile dieselbe fahlgelbliche Färbung, doch finden sich an dem zweiten Theile seiner Unterseite einige längere und stärkere, ziemlich borstenartige schwarze Haare. Der vorlelzte Bauchabschnitt des männlichen Hinlerleibs trägt an seinem Hinterrande zwei nicht sehr weit von einander entfernte Zapfen und der lelzte jederseits ein kleines Zäpfchen. Hüften schwarz, graulich bestäubt, mit fahlgelblicher Behaarung; die stärkeren, mehr borstenartigen Haare an Ende der vordersten und an der Aufsenseite der hinleren meist schwarz. Beine lang, glänzend schwarz, mit kurzer schwarzer Behaarung; Schenkel ziemlich kräftig aber nicht eigentlich verdickt, die hintersten von ansehnlicher Länge; die Unterseite der Vorderschenkel nur gegen ihr Ende bin sparsam beborstet, diejenige der Mittel- und Hinterschenkel ihrer ganzen Länge nach mit zahlreichen schwarzen Borsten von geringer und ungleicher Länge besetzt. Die Schienen sind in ihrer Färbung etwas veränderlich; bei den dunkelsten Exemplaren zeigt 
sich nur an der Basis der Hinterschienen und auch hier oft nur auf der Oberseite eine bräunlichgelbe oder rothgelbe Färbung; bei den meislen Stücken breitet sich diese Färbung auf der Oberseite der Hinterschienen sehr weit, nicht selten bis zum letzten Viertel hin aus, während sie auf der Unterseite auf die Schienenwurzel beschränkt bleibt und hier nie so hell wie auf der Oberseite ist; bei solchen Stücken zeigt sich an der Basis der Mittelschienen eine ähnliche Färbung, welehe aber stets eine geringere Ausdehunng als diejenige der Hinlerschienen hat; nur bei einzelnen Stücken ist die helle Färbung an den hinteren Schienen noch ausgedehnter und bei solchen zeigl sich dann zuweileu auch an der Wurzel der Vorderschienen eine mehr oder weniger deutlich auftretende helle Färbung. Alle Schienen sind auf der Oberseite sparsam mit kurzen schwarzen Borslen besetzt; die Hinterschienen sind gerade und von gleichmälsiger Stärke; auf der Unterseite derselben findel sich kurze, bei dem Männchen mehr abstehende und mehr wimperartige Behaarung. Klauen und Pulvillen des Männchens nur wenig gröfser als die des Weibchens. Schwinger blafsgelblich. Flügel sehr lang und verhälınilsmäfsig schmal, glasartig, nur mit äufserst schwacher Spur von graulicher Trübung, an der Basis mit einer eben so schwachen Spur von gelblicher Färbung; Adern dunkelbraun, in der Nähe der Basis lehumgelblich; das wenig bemerkbare grauliche Randmal ist sehr schmal; erste Submarginalzelle nur von näfsiger Breite; die drilte Längsader an ihrem Ende sanfı nach hinten gebogen; Vorderast derselben gekrümmt und ziemlich schief, verhältnifsmäfsig weit vor ihrem Ende eingesetzt, so dafs die zweite Submarginalzelle ziemlich grol's ist; erste Hinterrandszelle gegen ihr Ende hin nur wenig verbreitert; Discoidalzelle gröfser als bei den vorhergehenden Arten und an ihrem Ende viel weniger schief. - Körperlänge $2 \frac{3}{4}-2 \frac{5}{6}$, Flügellänge $3 \frac{1}{6}-3 \frac{5}{T^{\frac{5}{2}}} \mathrm{Lin}$.

Anmerkung. Walker zählt Emp. Bislortae als englische Arl auf; seine Beschreibung läfst vermuthen, dals er eine von Emp. truncal a ganı verschiedene Art mit diesem Namen belegt haben möge.

4. Emp. lucida Zett. schliefst sich nahe an Emp. Iruncata an, zeigt aber doch einige bemerkenswerthe plastische Eigenthümlichkeiten. Sie ist von elwas gestreckterem Körperbaue, ganz und gar glänzend schwarz und durchweg schwarz behaart. Die Augen des Männchens, welche bei Emp. truncala nur slark genähert sind, stofsen bei ihr auf der Stirn wirklich zusammen. Fühler und Rüssel von ähnlichem Baue wie bei Emp. Iruncata, das dritte Glied der letzteren aber erheblich breiter; der Fühlergriffel noch etwas kürzer aber ziemlich schlank. Stirn mit kurzer Behaa- 
rung; die Behaarung des Scheitels und des oberen Theils des Hinterkopfs weniger borstenartig. Die grauweifsliche Bestäubung des Thoraxrückens ist sehr dünn und deshalb wenig bemerklich; man bemerkt sie nur dann deutlich. wenn man den Thoraxrücken von vorn ber und in sehr schräger Richtung betrachtet; die Striemen erscheinen dann glänzend schwarz. Die Behaarung des Thoraxrückens ist viel dichter als bei allen vorhergehenden Arlen und auch auf der Mittellinie vorhanden, zart, bei dem Männchen ziemlich lang, bei dem Weibchen ziemlich kurz. Die schwarze Behaarung des Hinterleibs ist von ähnlicher Beschaffenheit wie bei Emp. truncata, nur nicht so lang, auch nicht so gleichmäfsig, da die Haare am Hinterrande der einzelnen Abschnitte eine gröfsere Stärke und Länge besilzen. Hypopygium wie bei den anderen Arten dieser Gruppe; der vorletzle Bauchabsehnitl des Männchens hat an seinem Hinterende nicht nur zwei warzenförmige Höcker, sondern auch eine ziemliche Anzah! gekrümmter schwarzer Borsten. Beine ganz schwarz, schlanker als bei Emp. truncata; die Schenkel bei weitem nicht so kräftig; Unterseite derselben nur sparsam beborslet, bei dem Weibchen noch erheblich sparsamer als bei dem Männchen; die Vorderschenkel sind bei diesem nur am Ende der Unterseite mit etlichen schwachen Borstchen besetzt. Die Borslen auf der Oberseite der Schienen sind schwach, aber länger und zahlreicher als bei Emp. truncata. Hinterschienen des Männchens auf der Unterseite mit kurıer, mälsig abstehender, dichter Behaarung; die Schienen des Weibchens baben daselbst äufserst kurze Behaarung. Klanen und Pulvillen des Männchens wenig gröfser als die des Weibchens. Flügel verhältnifsmäfsig merklich kürzer als die der Emp. truncala, die des Männchens fast glasartig mit deutlichem bräunlichem Farbenton, die des Weibchens mit auffallender bräunlicher Trübung; Adern dunkelbraun, in der Nähe der Basis gelbbräunlich; das schmale Randmal bräunlich; der Aderverlauf ähnelt dem der Emp. truncata sehr, nur ist die Discoidalzelle etwas kleiner und etwas schiefer. Als Vaterland sind bisher nur Skandinavien und England bekannt; ich selbst habe noch keine englischen Exemplare gesehen. Die Körperlänge beträgt $3-3 \frac{7}{\frac{1}{2}}$, die Flügellänge $3-3 \frac{1}{3}$ Lin.

5. Emp. longipennis nov. sp. aus dem westlichen Sibirien ist mir nur im weiblichen Geschlechte bekannt. Sie ist der Emp. lucida sehr nahe verwandt und stimmt im Körperbaue, wie in der Färbung und in der Art der Behaarung sehr mit ihr überein. Sie erscheint fast noch glänzender schwarz. Der Fühlergriffel ist noch etwas kürzer, aber dicker. Die Bestäubung des Thoraxrückens ist so dünn, dafs sie selbst auf den Inlerstitien nur schwer wahrzu- 
nehmen ist. Die Behaarung des ganzen Körpers ist, wie bei Emp. lucida, durchweg schwarz. Die Schwinger gelb. Das auffallendste Unterscheidungsmerkmal bilden die Flügel, welche verhältnifsmäs. sig länger und glashell sind; die Färbung der Flügeladerh ist der bei Emp. lucida vorhandenen ähnlich, doch etwas heller, namentlich fällt in der Nähe der Flügelwurzel die gelbe Färbung der Flügeladern viel mehr in das Auge; die Discoidalzelle ist gröfser als bei Emp. lucida und am Ende weniger schief, so dafs die Flügel in verhältnifsmäfsiger Länge, Gestalt, Aderverlauf und Färbung denen der Emp. truncala viel näher kommen, als denen der Emp. lucida. Körperlänge $3 \frac{5}{12}$, Flügellänge $3 \frac{2}{3} \mathrm{Lin}$.

Der systematische Platz der Emp. longipennis ist zwischen Emp. truncata und Emp. lucida.

Die fünf in gegenwärtigen Verwandtschaftskreis gehörigen Arten lassen sich in folgender Weise ordnen und unterscheiden.

I. Oculi maris inter se distantes; media thoracis linea nuda.

1. Emp. nitida Meig. $\delta$ et ㅇ. - Nigra, apice femorum tibiisque rufo-testaceis, his apicem versus plerumque obscurioribus, femoribus posticis valde incrassatis, alis maris hyalinis, foeminae basim versus fuscis. - Long. corp. $2 \frac{1}{6}-2 \frac{1}{3}$ lin., Long. al. $2 \frac{1}{3}-2 \frac{1}{2}$ lin.

Syn. Empis nitida Il eigen, Syst. Beschr. III. 39. 43.

2. Emp. monticola m. ơ et \&. - Nigra, pedibus obscure rufis, femoribus posticis modice incrassutis, alis subhyalinis, dilutissime subfuscis. - Long. corp. $2 \frac{1}{3}-2 \frac{7}{1} \frac{7}{2}$ lin., Long. al. $2 \frac{1}{1} \frac{1}{2}-3 \frac{1}{3}$ lin.

3. Emp. truncata Meig. $\sigma^{\circ}$ et + . - Nigra, tibiis posticis vel. posterioribus superne et in basi rufis, posticis non incrassatis, alis hyalinis. - Long. corp. $2 \frac{3}{4}-2 \frac{5}{6}$ lin., Long. al. $3_{6}^{\frac{1}{6}}-3_{\frac{5}{2}}$ lin.

Syn. Fmp. truncata Ml eigen, Syst. Beschr. III. 31, 27.

?Emp. Bistortae Meigen, Syst. Beschr. III. 29. 22.

II. Oculi maris contigui; media thoracis linea non nuda.

4. Emp. longipennis m. ㅇ. - Tota nigra, alis elongalis, hyalinis. - Long. corp. $3 \frac{5}{12}$ lin., Long. al. $3 \frac{2}{3}$ lin. -

5. Emp. lucida Zett. o' et 우 - Tota nigra, alis non elongatis, dilute subfuscis. - Long. corp. $3-3 \frac{7}{12}$ lin., Long. al. $3-3_{3}^{1}$ lin.

Syn. Emp. lucida Zetterstedt, Ins. Lapp. 561. 3.

Emp. lucida Zettersted t, Dipt. Scand. I. 373. 5. 


\section{$2 \mathrm{BHL}$ Biodiversity Heritage Library}

Loew, H. 1868. "Ueber Empis nitida Meig. und die ihr verwandten Arten." Berliner entomologische Zeitschrift / herausgegeben von dem Entomologischen Vereine in Berlin 12(2), 231-240. https://doi.org/10.1002/mmnd.18680120207.

View This Item Online: https://www.biodiversitylibrary.org/item/34406

DOI: https://doi.org/10.1002/mmnd.18680120207

Permalink: https://www.biodiversitylibrary.org/partpdf/210126

\section{Holding Institution}

Smithsonian Libraries

\section{Sponsored by}

Smithsonian

\section{Copyright \& Reuse}

Copyright Status: Public domain. The BHL considers that this work is no longer under copyright protection.

This document was created from content at the Biodiversity Heritage Library, the world's largest open access digital library for biodiversity literature and archives. Visit BHL at https://www.biodiversitylibrary.org. 